Mon. Not. R. Astron. Soc. 000, 18 (2019) Printed 9 October $2019 \quad$ (MN LATEX style file v2.2)

\title{
Silicon and iron dust in gamma-ray burst host galaxy absorbers
}

\author{
T. Zafar ${ }^{1 \star}$, K. E. Heintz ${ }^{2}$, A. Karakas ${ }^{3}$, J. Lattanzio ${ }^{3}$, A. Ahmad ${ }^{1}$ \\ 1 Australian Astronomical Optics, Macquarie University, 105 Delhi Road, North Ryde, NSW 2113, Australia \\ 2 Centre for Astrophysics and Cosmology, Science Institute, University of Iceland, Dunhagi 5, 107 Reykjavik, Iceland \\ 3 Monash Centre for Astrophysics, School of Physics and Astronomy, 10 College Walk, Monash University 3800, Australia
}

9 October 2019

\begin{abstract}
Depletion studies provide a way to understand the chemical composition of interstellar dust grains. We here examine 23 gamma-ray bursts (GRB) optical afterglow spectra (spanning $0.6 \leqslant z \leqslant 5.0$ ) and compare their silicon and iron dust-phase column densities with different extinction curve parameters to study the composition of the interstellar dust grains in these high-redshift GRB host galaxies. The majority of our sample (87\%) show featureless extinction curves and only vary in shape. We observe strong correlations (with $>96 \%$ significance) between the total-to-selective extinction, $R_{V}$, and the dust-phase column densities of Si and Fe. Since a large fraction of interstellar iron is locked in silicate grains, this indicates that high $\mathrm{Si}$ and $\mathrm{Fe}$ depletion leads to an increase in the fraction of large silicate grains and vice versa. This suggests that silicates play a vital role to induce the entire extinction at any wavelength. On the other hand, the far-UV extinction is usually attributed to the presence of small silicates. However, we find no trend between the far-UV parameter of the extinction curve, $c_{4}$, and the abundance of $\mathrm{Si}$ and $\mathrm{Fe}$ in the dust phase. We, therefore, propose that the far-UV extinction could be a combined effect of small (probably nanoparticles) dust grains from various species.
\end{abstract}

Key words: Galaxies: high-redshift - ISM: dust, extinction - Gamma rays: bursts

\section{INTRODUCTION}

Dust is an essential component of the interstellar medium (ISM) of galaxies (Dorschner \& Henning 1995) and is strongly linked with star formation (e.g., Cortese et al. 2012; Corre et al. 2018). It is therefore important to understand the chemical composition of interstellar dust grains. The interstellar dust grains are primarily made up of $\mathrm{O}, \mathrm{C}, \mathrm{Si}, \mathrm{Mg}$, and Fe elements (De Cia et al. 2016) which are introduced into the ISM during stellar evolution via stellar winds or at the end of star's life. Depletion studies of highly abundant species, i.e. silicon and iron, provide direct evidence that refractory elements go in and out of the dust-phase in the ISM (Jenkins 1987, 2009). These refractory elements are introduced into the ISM by stars during their stellar evolution or at the end of the life of a star and then completely condense into a solid form. Particularly silicon makes silicates by combining with different elements and may comprise up to $70 \%$ of the total dust grains mass in the local ISM (Weingartner \& Draine 2001). Silicate and carbona-

* e-mail:tayyaba.zafar@mq.edu.au ceous grains then evolve through many cycles of accretions, shattering, coagulation, and erosion happening in the ISM.

The Milky Way, Large and Small Magellanic Clouds (LMC and SMC) exhibit distinct extinction curves Cardelli et al. 1989; Pei 1992; Gordon et al. 2003; Valencic et al. 2003; Sofia et al. 2005; Fitzpatrick \& Massa 2007), showing large variations in the strength of the $2175 \AA$ extinction bump and the slope of the induced extinction curve. Stecher (1965) first discovered the $2175 \AA$ extinction bump in the extinction curves of the Milky Way. The feature becomes weaker in the LMC and SMC display a featureless extinction curve with a steep rise into the UV. The $2175 \AA$ bump has been attributed to absorption by carbonaceous dust grains produced by star formation (Draine 2003). The slope of the extinction curve is characterised by the total-to-selective extinction parameter, $R_{V}$. In the Milky Way, $R_{V}$ parameter ranges from $\sim 2-5$ (Fitzpatrick \& Massa 2007), where a small value of $R_{V}$ corresponds to a steep extinction curve. Large average dust grains therefore produce a 'flatter' extinction curve, resulting in large values of $R_{V}$ and vice versa Valencic et al. 2004).

Gamma-ray bursts (GRBs) are powerful probes 
to study the ISM of high-redshift galaxies (e.g., Prochaska et al. 2007; de Ugarte Postigo et al. 2012). At the same time, because of their simple power-law spectra, GRBs provide a unique tool to study the absolute extinction curves of their environments (e.g. Kann et al. 2006; Greiner et al. 2011; Schadv et al. 2012; Zafar et al. 2011b; Covino et al. 2013; Zafar et al. 2018c). Comparing extinction curve parameters with depletion of various refractory elements can provide us clues to the composition and distribution of interstellar dust grains in GRB host galaxy environments. For example, it has previously been found that the strength of the $2175 \AA$ extinction bump observed in GRB and quasar absorbers is correlated with the amount of neutral carbon (Krühler et al. 2008; Elíasdóttir et al. 2009; Perley et al. 2011; Zafar et al. 2012; Ledoux et al. 2015; Ma et al. 2018; Heintz et al. 2019a). Recently, Heintz et al. (2019b) also found an additional positive trend between the bump strength and $R_{V}$, so in combination with the relation to the amount of neutral carbon this suggests that the $2175 \AA$ bump is produced by large dust grains and molecules in the cold and molecular gas-phase of the ISM.

From the observations of 16 Galactic sightlines, Haris et al. (2016) claimed that that the silicon depletion correlates with the $2175 \AA$ bump and the far-UV extinction rise. However, more recently Mishra \& Li (2017) found that the bump strength correlates with carbon abundance instead of silicon and there is no correlation of far-UV extinction with either silicon or carbon abundance using a silicate-graphite model on a larger sample. This discrepancy could be related to the different methods adopted for determining the silicon dust depletion and usage of different interstellar abundances of silicon in the two studies. The depletion of various elements and its connection to dust and metals has also been studied previously in GRBs and quasar absorbers (e.g., Savaglio \& Fall 2004; Vladilo et al. 2006, 2011; De Cia et al. 2013; Zafar \& Watson 2013; De Cia et al. 2016; De Cia 2018; Zafar \& Møller 2019). For GRBs, Heintz et al. (2019b) already compared carbon against bump strength and $R_{V}$. GRBs usually exhibit featureless extinction curves (e.g. Kann et al. 2006; Greiner et al. 2011; Schadv et al. 2012; Covino et al. 2013; Zafar et al. 2018c). We here aim to compare for the first time silicon and iron depletion against different extinction curve parameters to study the chemical composition of dust in the average GRB environment further.

We carefully selected a sample of GRB afterglows with measurements of extinction curve parameters using the parametric dust model of Fitzpatrick \& Massa (1990) and with available silicon and/or iron abundances. In $\S 2$ we present our sample and methods used for investigation. In $\S 3$ we provide the results together with our discussion. Conclusions are given in $\S 5$. Throughout the paper, given errors are $1 \sigma$ unless stated otherwise.

\section{SAMPLE SELECTION AND METHOD}

For consistency, we included GRBs in our sample that have been examined using the same dust model to fit their spectral energy distributions (SEDs). Also for these
GRBs, we selected the studies where the GRB optical extinction is derived from simultaneous SED fitting to $\mathrm{X}$ ray-to-optical/NIR data using either a single or broken power-law. This way, the intrinsic slope of the GRB is constrained by the X-ray data, providing a reliable method to determine the extinction curve of the burst. We, therefore, used GRBs from Zafar et al. (2018c, b) and Heintz et al. (2019b) using a freeform parametric dust model of Fitzpatrick \& Massa (1990). See Zafar et al. (2018c) for more details on the extinction model. In the following we only consider the SED-derived extinction curve parameters $R_{V}, c_{4}$ (far-UV parameter), and $A_{V}$ for the GRBs in our sample. We first searched in the literature for the refractory element (silicon: Si II and/or iron: Fe II) abundances of these GRBs. We found that only 16 GRBs have published abundances of these refractory elements to enter into our sample. In addition, we included 7 more GRBs with extinction curve measurements from Zafar et al. (in prep) with available measurements of $\mathrm{Si}$ and/or Fe abundances. A consistent method to Zafar et al. (2018c) is used in Zafar et al. (in prep), the latter including the entire X-shooter GRB afterglow legacy sample presented in Selsing et al. (2019). This make up a total of $23 \mathrm{GRBs}$ in our sample within the redshift range of $0.6 \leqslant z \leqslant 5.0$ (see Table 1 ).

We then looked for volatile element abundances (sulphur: S II and/or zinc: Zn II) for these 23 cases to make pairs with refractory element abundances to derive depletion (i.e. $\delta_{X}=\log (N(X) / N(Y))-\log (X / Y) \odot$; where $X=$ refractory and $Y=$ volatile element). However, we find only 8 cases with sulphur and 17 cases with zinc abundances measurements.

We derived dust-phase column densities (i.e. $\left.N(X)_{\text {dust }}=\left(1-10^{\delta_{X}}\right) N(Y)(X / Y)_{\odot}\right)$ of $\mathrm{Si}$ and $\mathrm{Fe}$ for our GRBs where abundance pairs are available using the depletions. For the remaining GRBs without abundance pairs, we instead used the tight dust-to-metals correlation of Zafar \& Møller (2019). This relation was derived by using the observations of $93 \mathrm{GRB}$ afterglows and quasar absorbers $(z \approx 0.4-6.3)$. The GRBs included in their sample are in the range of $z \sim 1.1-6.3$ where $A_{V}$ is derived from the afterglow SED fitting and metal abundances are obtained from absorption line analysis. Using the Zafar \& Møller (2019) relation, we first determine the 'total' column density of refractory elements. This is done by using $A_{V}$ values for each case and the estimated correlation of $A_{V} / N(\mathrm{H} \mathrm{I}) \times 10^{\left[\mathrm{Si}_{\text {tot }}, \mathrm{Fe}_{\text {tot }} / \mathrm{H}\right]}=(4.91 \pm 0.98) \times 10^{-22}$. The dust-phase column density of $\mathrm{Si}$ and $\mathrm{Fe}$ is then calculated by subtracting the observed column density of refractory elements from the total (since $N(\mathrm{X})_{\text {tot }}=$ $\left.N(\mathrm{X})_{\text {dust }}+N(\mathrm{X})_{\text {obs }}\right)$. The above mentioned correlation, instead of the direct relation of $A_{V}$ and dust-phase column density of the refractory elements, is used as it is the strongest relation, matches to the Local Group relation, and provides an opportunity of using the observed refractory element abundances.

The majority of the spectra in our sample have been obtained with the VLT/X-shooter with medium spectral resolution where hidden saturation could be a concern and a narrow $3-5 \mathrm{kms}^{-1}$ component may not be resolved. The column densities are derived by fitting Voigt profiles to multiple transitions of the ions simultaneously where weak lines provide the accurate measurements of column densi- 


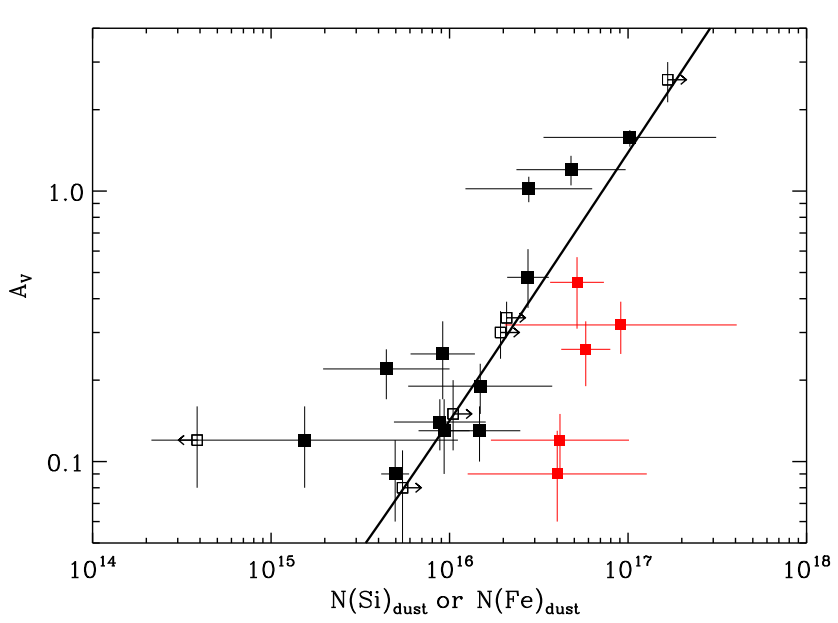

Figure 1. Extinctions as a function of Si (black) and Fe (red) dust-phase column densities for GRBs. The $N(\mathrm{Fe})_{\text {dust }}$ is only considered for the cases where there is no $N(\mathrm{Si})_{\text {dust }}$ measurement available. The solid line represents the Pearson linear correlation with a best-fit slope of $y / x=(1.39 \pm 1.03) \times 10^{-17}$. The filled squares are measurements while the open squares are limits.

ties. The studies from where abundances are included in our sample have paid attention while deriving abundance measurements. A narrow hidden component could in principle underestimate the element column density by $\sim 0.1$ dex (Zafar et al. 2011a; Rafelski et al. 2012; Cucchiara et al. 2015; Wiseman et al. 2017) and hence overestimate the dust-phase column density. Our error bars on the dust-phase column densities are larger than 0.1 dex so this issue will not affect our results significantly. Moreover, our chosen volatile elements $\mathrm{S}$ and $\mathrm{Zn}$ are also mildly depleted, particularly, in the presence of high molecular fraction. We refer the reader to the discussions in De Cia et al. (2016) and Zafar \& Møller (2019). We have only 4 cases (GRB 120815A, GRB 120909A, GRB 121024A, and GRB 141109A) reported with the presence of molecules (see Bolmer et al. 2019) and for these the dust-phase abundances of $\mathrm{Si}$ and $\mathrm{Fe}$ may be slightly underestimated.

The relation between the visual extinction. $A_{V}$, and dust-phase column density of refractory elements has been studied previously for quasar absorbers (Vladilo et al. 2006) and GRB systems (De Cia et al. 2013). We here compared our dust-phase abundances of $\mathrm{Si}$ and $\mathrm{Fe}$ against other extinction parameters, namely $1 / R_{V}$ and the far-UV component $\left(c_{4}^{\prime}=c_{4} / R_{V}\right)$. We consider $c_{4}^{\prime}$ here because in the parametrization of Fitzpatrick \& Massa (1990) all the $c$ parameters are normalised by $R_{V}$. Therefore, $c_{4}^{\prime}$ better describe the shape of the entire far-UV component.

\section{RESULTS AND DISCUSSIONS}

Fig. 1 shows that for our sample, extinction is linearly correlated with the dust-phase column density of silicon or iron. This trend is already known for GRBs and quasar absorbers (Vladilo et al. 2006; Zafar \& Møller 2019). We fit a Pearson linear relation to our data and find that the data fit well (significance $\alpha>99 \%$ and correlation coefficient $\rho=+0.83$ ) with a slope of $y / x=(1.39 \pm 1.03) \times 10^{-17}$. This is consis- tent with the relation found by Zafar \& Møller (2019) and expected as some of the dust phase column densities are computed using the $A_{V}$ values and 13 out of 23 GRBs are included in both studies. This suggests that, overall, the dust grains producing the observed extinction are linked to the abundance of $\mathrm{Si}$ and $\mathrm{Fe}$ in the dust phase.

\section{1 $R_{V}$ against dust-phase $\mathrm{Si}$ and $\mathbf{F e}$}

We compared dust-phase column densities of $\mathrm{Si}$ and $\mathrm{Fe}$ for GRBs with $R_{V}^{-1}$ and far-UV extinction component, $c_{4}^{\prime}$ (see Fig. 2). As the top panels of Fig. 2 show, $R_{V}^{-1}$ anticorrelates with both $N(\mathrm{Si})_{\text {dust }}$ and $N(\mathrm{Fe})_{\text {dust }}$. A linear regression analysis of $R_{V}^{-1}$ versus $N(\mathrm{Si})_{\text {dust }}$ and $N(\mathrm{Fe})_{\text {dust }}$ for the GRBs in our sample yields a Pearson's correlation coefficient $\rho=-0.63$ (significance $\alpha \approx 96 \%$ ) and $\rho=-0.48$ (significance $\alpha \approx 96 \%$ ), respectively. Since $R_{V}$ is the absolute extinction to color excess ratio $\left(R_{V}=A_{V} / E(B-V)\right)$, and it correlates with the shape of extinction curves (Cardelli et al. 1989), this relation suggests that the Si and Fe dust components correlate well with the extinction over the entire wavelength range from the visible to the far-UV. The value of $R_{V}$ is directly related to the size of the dust grains: Large $R_{V}$ values are produced by larger grains in the environment and small $R_{V}$ values are characterised by smaller grains. Low $R_{V}$ sightlines produce very steep extinction curves in the UV/optical and comprise a large fraction of small dust grains and vice versa (Valencic et al. 2004). Our results suggest that with an increase in $\mathrm{Si}$ and Fe depletion, the average grain sizes will also increase in the ISM. This is consistent with most of the $\mathrm{Si}$ and Fe being depleted from the gasphase and accreting onto dust grains. In particular, Draine (1990) proposed that in dense environments with high depletion, small grains coagulate onto large grains. It is also worth noting that shattering during grain-grain collisions in shock waves can return dust grain mass from large to small grains (Weingartner \& Draine 2001).

Previously, Haris et al. (2016) reported a weak trend (with a Pearson's correlation coefficient $\rho=-0.13$ and significance $\alpha \approx 37 \%$ ) between $R_{V}$ and Si depletion. We plotted their data in Fig. 2 in grey colour, however, the Haris et al. (2016) data are not included in our correlation analysis. We attempted to compare their results with our work. We used their Tables $3-4$ to compare $R_{V}$ with the dust-phase column density of $\mathrm{Si}$ for the Galactic sightlines. This is done to make their work comparable to this study. Moreover, extinction is generated by dust particles and instead of comparing relative measurement of refractory element in dust (depletion) it is useful to compare column densities of their atoms in the dust. Their $\log N(\mathrm{Si})_{\text {dust }}$ ranges from $\sim 16.5-17.3$ and $R_{V}$ ranges from $\sim 2-6$. A linear regression analysis of their data yields a Pearson's correlation coefficient $\rho=-0.57$ (significance $\alpha \approx 98 \%$ ), consistent with our findings. Top-left panel of Fig. 2 also shows that the trend is more apparent at $\log N(\mathrm{Si})_{\text {dust }}>16$ as seen in the data of Haris et al. (2016). Our relation between $R_{V}^{-1}$ and silicon and iron in dust suggests that dust grain size varies as a function of the amount of $\mathrm{Si}$ and $\mathrm{Fe}$ in the dust-phase. 


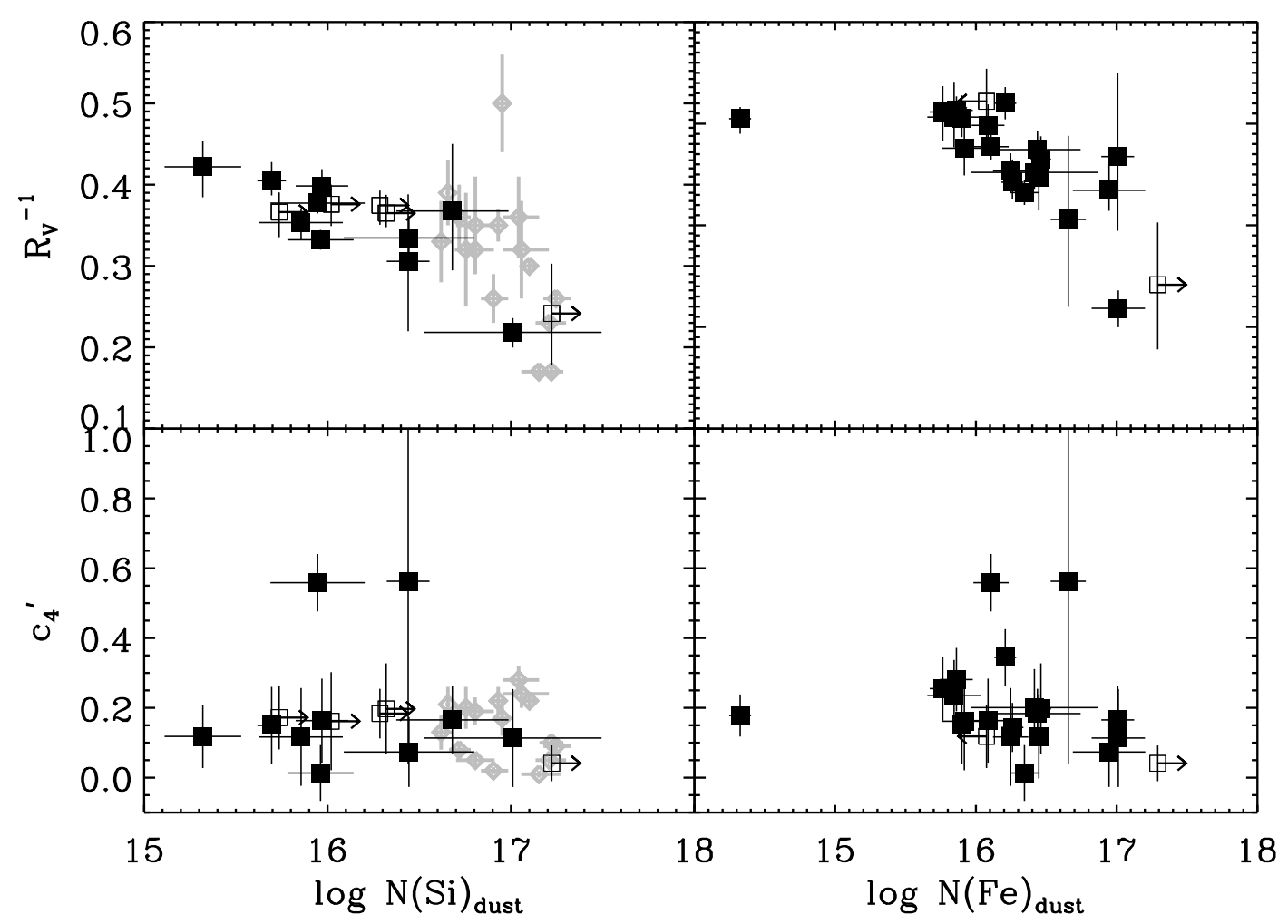

Figure 2. Inverse total-to-selective extinction $\left(R_{V}^{-1} ;\right.$ top panels $)$ and far-UV component $\left(c_{4}^{\prime} ;\right.$ bottom panels $)$ compared against dust-phase column densities of silicon and iron for GRB afterglows. The filled squares represent measurements and open squares indicate limits. The Galactic silicon dust-phase column densities from Haris et al. (2016) are shown as gray diamonds for a comparison.

\subsection{Far-UV rise against dust-phase Si and Fe}

At $\lambda^{-1}>5.9 \mu \mathrm{m}^{-1}$ of the extinction curve, $c_{4}^{\prime}$ only measures the far-UV non-linear rise and is not representative of the entire UV extinction. We observe no trend between $c_{4}^{\prime}$ with either $N(\mathrm{Si})_{\text {dust }}$ or $N(\mathrm{Fe})_{\text {dust }}$ (see bottom panels of Fig. 2). A linear regression analysis of $c_{4}^{\prime}$ versus $N(\mathrm{Si})_{\text {dust }}$ and $N(\mathrm{Fe})_{\text {dust }}$ for our sample yields a Pearson's correlation coefficient $\rho=-0.07$ (significance $\alpha \approx 16 \%$ ) and $\rho=-0.12$ (significance $\alpha \approx 36 \%$ ), respectively. This is in contrast to the findings of Haris et al. (2016) who claimed with just a weak Pearson's correlation coefficient $(\rho=-0.32)$ that silicon depletion correlates with the far-UV rise. As in 3.1 we compared their $N(\mathrm{Si})_{\text {dust }}$ of Galactic sightlines with $c_{4}^{\prime}$ values resulting in an even weaker Pearson's correlation coefficient $\rho=-0.17$ (significance $\alpha \approx 47 \%$ ), consistent with our results. In this parametrization of the extinction curve, $c_{4}^{\prime}$ represents the population of small grains. Previously, extinction curve models suggested that small silicate grains are responsible for the far-UV rise (Mathis et al. 1977; Draine \& Lee 1984; Weingartner \& Draine 2001; Clayton et al. 2003). However, Mishra \& Li (2017) fitted the observed Galactic sightlines with a silicate-graphite dust model and reported that neither silicate nor carbonaceous dust correlates with the farUV extinction. The far-UV extinction may therefore originate from a combined effect of both small carbon and silicate grains. A larger sample of more high quality measurements of extinctions and silicon and iron column densities will provide an even better understanding of the relation between the dust-phase refractory element abundances and ultraviolet extinction parameters.

We also note that both Haris et al. (2016) and Mishra \& Li (2017) conducted their studies using Galactic sightlines usually exhibiting $2175 \AA$ bump in their extinction curves, the bump being attributed to carbonaceous dust grains (Draine 2003). Still, both studies have obtained discrepant results, likely due to the different methods used to estimate dust abundances (i.e., $[\mathrm{Si} / \mathrm{H}]_{\text {dust }}=[\mathrm{Si} / \mathrm{H}]_{\mathrm{ISM}}-$ $[\mathrm{Si} / \mathrm{H}]_{\text {gas }}$. To derive silicon depletion, Haris et al. (2016) assumed the interstellar abundance of silicon to be solar. On the other hand, Mishra \& Li (2017) considered three approaches to derive dust-phase abundances of $\mathrm{Si}$ : $i$ ) considering two sets of interstellar abundances: the protosolar abundance of Lodders (2003) and the B-star abundances of Przybilla et al. (2008), ii) using Kramers-Kronig Relation of Purcell (1969), and iii) modelling the observed extinction curves using a silicate-graphite model. Additionally, they reported the results obtained from extinction curve modelling of the same Galactic sightlines.

In our study, out of the total 23 GRB afterglows only three GRB extinction curves exhibit the $2175 \AA$ extinction bump (i.e. GRB 070802 Elíasdóttir et al. 2009; GRB 080607 Perley et al. 2011; and GRB 180325A Zafar et al. 2018a) Also we have used volatile element abundances to derive depletions. The difference in the methods of deriving depletions, types of extinction curves and hence environments and dust composition of the grains present in these environments may cause the discrepant results. We argue that 
Table 1. GRB afterglow data used to estimate N(Si, Fe $)_{\text {dust }}$. The columns are given as: (1) GRB name, $(2)$ extinction $\left(A_{V}\right),(3)$ redshift, (4-7) observed Si II, Fe II, S II, Zn II column densities, (8) far-UV parameter $c_{4}$, (9) total-to-selective extinction $R_{V}$, and (10) References (where the first reference is for dust extinction measurement and second reference(s) is for metal column density measurement).

\begin{tabular}{|c|c|c|c|c|c|c|c|c|c|}
\hline GRB & $\begin{array}{l}A_{V} \\
\text { mag }\end{array}$ & $z$ & $\begin{array}{c}\log \mathrm{N}(\mathrm{Si} \text { II }) \\
\mathrm{cm}^{-2}\end{array}$ & $\begin{array}{c}\log \mathrm{N}(\mathrm{Fe} \text { II }) \\
\mathrm{cm}^{-2}\end{array}$ & $\begin{array}{c}\log \mathrm{N}(\mathrm{S} \text { II }) \\
\mathrm{cm}^{-2}\end{array}$ & $\begin{array}{c}\log \mathrm{N}(\mathrm{Zn} \mathrm{II}) \\
\mathrm{cm}^{-2}\end{array}$ & $c_{4}$ & $R_{V}$ & Refs. \\
\hline 061121 & $0.46_{-0.15}^{+0.11}$ & 1.315 & $\cdots$ & $16.20 \pm 0.03$ & $\cdots$ & $13.76 \pm 0.06$ & $0.34 \pm 0.12$ & $2.88_{-0.27}^{+0.23}$ & 1 \\
\hline 070802 & $1.20 \pm 0.15$ & 2.455 & $16.60 \pm 0.30$ & $16.10 \pm 0.10$ & $\ldots$ & $13.60 \pm 0.60$ & $0.45 \pm 0.09$ & $2.72_{-0.54}^{+0.61}$ & 1,2 \\
\hline 080605 & $0.48_{-0.11}^{+0.13}$ & 1.640 & $15.88 \pm 0.10$ & $14.66 \pm 0.11$ & $\cdots$ & $13.53 \pm 0.14$ & $1.84 \pm 0.50$ & $3.27_{-0.92}^{+0.88}$ & 1,3 \\
\hline 080607 & $2.58_{-0.45}^{+0.42}$ & 3.037 & $>16.34$ & $>16.70$ & $\cdots$ & $\cdots$ & $0.17 \pm 0.05$ & $4.14_{-1.09}^{+1.05}$ & 1,3 \\
\hline 090313 & $0.30 \pm 0.06$ & 3.374 & $>15.42$ & $15.08 \pm 0.30$ & $\cdots$ & $\cdots$ & $0.49 \pm 0.07$ & $2.67_{-0.17}^{+0.13}$ & 4,5 \\
\hline 090809 & $0.12 \pm 0.04$ & 2.737 & $16.15 \pm 0.07$ & $15.75 \pm 0.07$ & $15.85 \pm 0.60$ & $13.25 \pm 0.11$ & $0.62 \pm 0.09$ & $2.43_{-0.17}^{+0.15}$ & 3,6 \\
\hline 100219A & $0.14 \pm 0.03$ & 4.668 & $15.15 \pm 0.25$ & $14.73 \pm 0.11$ & $14.25 \pm 0.15$ & $\cdots$ & $1.48 \pm 0.08$ & $2.65 \pm 0.09$ & 3,4 \\
\hline $100418 \mathrm{~A}$ & $0.12 \pm 0.03$ & 0.624 & $\cdots$ & $15.62 \pm 0.10$ & $\ldots$ & $13.59 \pm 0.25$ & $0.68 \pm 0.09$ & $2.42_{-0.10}^{+0.08}$ & 4,7 \\
\hline $100901 \mathrm{~A}$ & $0.25 \pm 0.08$ & 1.408 & $15.96 \pm 0.17$ & $15.23 \pm 0.08$ & $\cdots$ & $13.52 \pm 0.07$ & $0.04 \pm 0.08$ & $3.01 \pm 0.11$ & 4,8 \\
\hline $111008 \mathrm{~A}$ & $0.12 \pm 0.04$ & 4.991 & $>16.04$ & $16.05 \pm 0.05$ & $15.71 \pm 0.09$ & $13.28 \pm 0.21$ & $0.44 \pm 0.06$ & $2.47_{-0.09}^{+0.07}$ & 3,4 \\
\hline $111107 \mathrm{~A}$ & $0.13 \pm 0.03$ & 2.893 & $15.87 \pm 0.20$ & $<14.70$ & $\ldots$ & $\ldots$ & $0.28 \pm 0.09$ & $2.37_{-0.21}^{+0.18}$ & 6,9 \\
\hline 120119A & $1.02 \pm 0.11$ & 1.729 & $16.67 \pm 0.35$ & $15.95 \pm 0.25$ & $\ldots$ & $14.04 \pm 0.25$ & $0.22 \pm 0.10$ & $2.99_{-0.18}^{+0.21}$ & 3,4 \\
\hline $120712 \mathrm{~A}$ & $0.08 \pm 0.03$ & 4.172 & $>14.6$ & $\cdots$ & $\cdots$ & $\cdots$ & $0.47 \pm 0.09$ & $2.73_{-0.23}^{+0.18}$ & 9,10 \\
\hline $120716 \mathrm{~A}$ & $0.32 \pm 0.07$ & 2.487 & $16.48 \pm 0.45$ & $15.65 \pm 0.45$ & $\cdots$ & $13.91 \pm 0.32$ & $0.57 \pm 0.11$ & $2.84_{-0.13}^{+0.21}$ & 6,11 \\
\hline $120815 \mathrm{~A}$ & $0.19 \pm 0.04$ & 2.360 & $16.34 \pm 0.16$ & $15.29 \pm 0.05$ & $16.22 \pm 0.25$ & $13.47 \pm 0.06$ & $0.82 \pm 0.08$ & $2.38 \pm 0.09$ & 3,4 \\
\hline 120909A & $0.09_{-0.03}^{+0.04}$ & 3.929 & $16.22 \pm 0.32$ & $15.20 \pm 0.18$ & $\ldots$ & $13.55 \pm 0.32$ & $0.58 \pm 0.10$ & $2.46_{-0.18}^{+0.21}$ & $6,9,11$ \\
\hline $121024 \mathrm{~A}$ & $0.26 \pm 0.07$ & 2.300 & $16.69 \pm 0.14$ & $15.82 \pm 0.05$ & $\ldots$ & $13.74 \pm 0.03$ & $0.42 \pm 0.07$ & $2.92_{-0.14}^{+0.19}$ & $4,9,12$ \\
\hline $130408 \mathrm{~A}$ & $0.22_{-0.05}^{+0.04}$ & 3.758 & $15.95 \pm 0.22$ & $15.52 \pm 0.11$ & $15.78 \pm 0.18$ & $12.87 \pm 0.16$ & $0.33 \pm 0.14$ & $2.83_{-0.17}^{+0.14}$ & 3,4 \\
\hline $140311 \mathrm{~A}$ & $0.15_{-0.04}^{+0.05}$ & 4.955 & $>14.70$ & $15.78 \pm 0.15$ & $15.48 \pm 0.12$ & $13.28 \pm 0.14$ & $0.43 \pm 0.14$ & $2.66_{-0.19}^{+0.17}$ & 9,10 \\
\hline $141028 \mathrm{~A}$ & $0.13 \pm 0.04$ & 2.333 & $14.26 \pm 0.13$ & $14.29 \pm 0.10$ & $\ldots$ & $12.38 \pm 0.33$ & $0.41 \pm 0.12$ & $2.51_{-0.14}^{+0.13}$ & 6,9 \\
\hline $141109 \mathrm{~A}$ & $0.34 \pm 0.05$ & 2.994 & $>15.60$ & $15.54 \pm 0.04$ & $15.81 \pm 0.05$ & $13.18 \pm 0.06$ & $0.54 \pm 0.13$ & $2.74_{-0.13}^{+0.17}$ & 6,9 \\
\hline $161023 \mathrm{~A}$ & $0.09 \pm 0.03$ & 2.710 & $15.21 \pm 0.05$ & $14.80 \pm 0.03$ & $14.85 \pm 0.03$ & $\ldots$ & $0.37 \pm 0.11$ & $2.47_{-0.11}^{+0.14}$ & 6,9 \\
\hline $180325 \mathrm{~A}$ & $1.58_{-0.12}^{+0.10}$ & 2.249 & $16.12 \pm 0.48$ & $16.68 \pm 0.18$ & $\cdots$ & $13.13 \pm 0.14$ & $0.52 \pm 0.14$ & $4.58_{-0.39}^{+0.37}$ & 13 \\
\hline
\end{tabular}

1: (Heintz et al. 2019b), 2: (Elíasdóttir et al. 2009), 3: (Zafar \& Møller 2019, and references therein), 4: (Zafar et al. 2018c), 5: (de Ugarte Postigo et al. 2010), 6: Zafar et al. (in prep), 7: (de Ugarte Postigo et al. 2018), 8: (Hartoog et al. 2013), 9: (Bolmer et al. 2019), 10: (Zafar et al. 2018b), 11: (Wiseman et al. 2017), 12: (Friis et al. 2015), 13: (Zafar et al. 2018a)

our results are more reliable for high redshift studies where featureless extinction curves are more typically observed.

\subsection{Dust grain composition and sizes}

Silicon and iron are highly depleted elements in the early Universe. Their high abundance ratios are linked to high star formation rates (Tinsley 1979). Nucleosynthesis yields of heavy elements also suggest a connection between iron and silicon abundance and Type II supernova (SN; Kobavashi et al. 2006; Kobavashi \& Nakasato 2011). Low and intermediate mass stars $\left(0.8<M_{\star} / M_{\odot}<8\right)$ during their asymptotic giant branch phase (Gail 2010), corecollapse SNe (Gall et al. 2011) and grain growth in the ISM by selective mantle accretion (Draine 2009) are considered as viable mechanisms for dust formation. Particularly, corecollapse $\mathrm{SNe}$ are believed to produce most heavy elements in a very short timescale.

In the Galactic disk, the fractions of $\mathrm{Si}$ and $\mathrm{Fe}$ in the gas-phase decreases by a factor of ten between the warm and cold neutral medium (CNM \& WNM; Savage \& Sembach 1996). Silicon is more depleted in the CNM, while the iron is more in the WNM and is an important component of interstellar dust because of its comparable abundance to $\mathrm{Mg}$ and Si (Zhukovska et al. 2016). Zhukovska et al. (2016), in their hydrodynamic simulation, attempted to explain the high depletion of Si in a Milky-Way like galaxy and find it to be a combination of accretion of $\mathrm{Si}$ on silicate grains in the CNM and efficient dust destruction by supernovae shocks in the diffuse gas. Most of the Si atoms have been locked up in silicate dust grains ( $\mathrm{Li}$ 2005). Based on condensation sequences, most of iron and magnesium are expected to condense in silicates and some $\mathrm{Al}, \mathrm{Na}$, and $\mathrm{Ca}$ might also be locked up in silicates (Tielens \& Allamandola 1986). The observed Fe depletion can be explained by the accretion of $\mathrm{Fe}$ onto dust grains (Dwek 2016). A large fraction of interstellar iron is, for example, locked in silicate grains as demonstrated by the in-situ studies of dust grains (Westphal et al. 2014; Altobelli et al. 2016). The depleted Fe can be in form of metallic iron (Schalen 1965) with no extinction features, in silicate lattice (Ossenkopf et al. 1992), as pure iron and $\mathrm{FeS}$ to the silicates (Min et al. 2007; Jones et al. 2013), as iron oxides to the silicates (Draine \& Henslev 2013), or in form of iron nanoparticles (Hensley \& Draine 2017; Gioannini et al. 2017; Bilalbegović et al. 2017). The high depletion of both $\mathrm{Si}$ and Fe can also be explained by nanosized grains (Zhukovska et al. 2018).

Silicates are an important component in every dust grain model of the ISM (e.g., Mathis et al. 1977; Clavton et al. 2003; Siebenmorgen et al. 2014; Zhukovska et al. 2016). The models show that the efficient growth of silicate iron dust commences in the cold diffuse ISM (Zhukovska et al. 2018). Silicate dust grain formation has also been identified in SN ejecta (e.g., Haenecour et al. 
2013; Sarangi \& Cherchneff 2015). Silicate dust in the ISM is found to be entirely amorphous (e.g., Kemper et al. 2004) and crystalline in addition to amorphous in protoplanetary disks (e.g., Sargent et al. 2009). De Cia et al. (2016) used an observational sample of 70 damped Ly $\alpha$ absorbers towards quasars at intermediate redshifts and reported that after oxygen, which is ten times more abundant, $\mathrm{Mg}$, $\mathrm{Fe}$, and $\mathrm{Si}$ are other important dust constituents. The similar abundances of $\mathrm{Mg}, \mathrm{Si}$, and $\mathrm{Fe}(\mathrm{Mg}: \mathrm{Si}: \mathrm{Fe} \sim 1)$ in dust indicate that amorphous silicates such as pyroxenes (e.g., $\mathrm{MgSiO}_{3}$ and $\mathrm{FeSiO}_{3}$ ) and iron oxides (e.g., $\mathrm{FeO}$ and $\mathrm{Fe}_{2} \mathrm{O}_{3}$ ) are dominant grain species, while silicates such as olivine (e.g., $\mathrm{Mg}_{2} \mathrm{SiO}_{4}$ and $\mathrm{Fe}_{2} \mathrm{SiO}_{4}$ ) might be less important (De Cia et al. 2016). Our results suggest that high Si and Fe depletion indicates the presence of more silicates in the ISM and variation in their sizes can alter the change in the shape of the individual featureless extinction curves.

On the other hand, carbonaceous dust grains are formed in the ISM when there is sufficient carbon available. When a large reservoir of metals is available, non-carbonaceous grains will form via grain growth in the ISM (Draine 2009). De Cia et al. (2016), in their study of dust composition, suggested that the presence of carbon or the grain size distribution are responsible for extinction curves shapes, although carbon is not included in their analysis. De Cia et al. (2016) reported that the main dust constituent is oxygen and up to $50 \%$ of oxygen is locked in silicate grains and iron oxides. Heintz et al. (2019b) and Mishra \& Li (2017) found a correlation between $R_{V}$ and carbon (gas and dust phase) suggesting carbon grains as the possible carrier of the $2175 \AA$ extinction bump. However, we find here that in addition to the abundance of carbon and the average grain size distribution, the amount of silicate grains can also alter the shape of the extinction curve. In our sample, $87 \%$ (20 out of 23 GRBs) show featureless extinction curves, which implies that silicates play a dominant role to induce the featureless extinction curve shape when carbon grains do not dominate the dust content.

The interstellar dust size distributions extends down to nanometer sizes. Nanoparticles recovered meteorites could be a realistic model for interstellar grains (Mautner et al. 2006). Still the modelling of nanoparticles is challenging where not only the effects of environment on the particles but also their return effects (photo-electric heating of gas, formation of molecules etc.) should be taken into account (Jones 2016). Smaller grains, like nano size silicate dust grains, may contribute to the far-UV extinction Draine 1995) and could be carriers of observed extended red emission (Zubko et al. 1999). Although the exact sizes of these nano grains cannot be constrained by the far-UV extinction (Wang et al. 2015), and since they are suggested to account for only $5 \%$ of total interstellar silicon, they have no significant effect on Si depletion (Li \& Mann 2012). Iron nanoparticles have also been proposed to partly contribute to the far-UV extinction (Henslev \& Draine 2017). Inclusion of iron nanoparticles within large silicate grains has been directly analysed (Westphal et al. 2014; Hilchenbach et al. 2016) and they are suggested to be a vital contributor to interstellar extinction (Köhler et al. 2014). Our results here suggest that the far-UV extinction could be a combined effect of small (or nano) dust grains from various species. Also high $\mathrm{Si}$ and $\mathrm{Fe}$ (since its large fraction is locked in silicates) depletion increases the fraction of large silicate grains in the environment and hence responsible for flatter extinction curves.

\section{CONCLUSIONS}

Understanding interstellar dust is important to study ISM and star-formation in galaxies. The attribution of the $2175 \AA$ bump of the extinction curve to the carbonaceous dust is well studied for GRBs and quasar absorbers (Zafar et al. 2012; Ledoux et al. 2015; Ma et al. 2018; Heintz et al. 2019a, b). We here compared extinction curve parameters to the Si and Fe dust-phase column densities to infer the composition of dust grains in the average GRB absorber population, showing typically featureless extinction curves. We analyzed a sample of $23 \mathrm{GRB}$ afterglow $(0.6 \leqslant z \leqslant 5.0)$ with available extinction curve parameters derived from X-ray-to-optical/NIR SED fitting and observed $\mathrm{Si}$ and Fe column densities. The majority of our sample $(87 \%)$ indeed do show featureless extinction curves. We then computed Si and Fe dust-phase column densities of these GRBs either estimating depletions using volatile element abundances ( $\mathrm{S}$ and $\mathrm{Zn}$ ) or using the dust-to-metals relation of Zafar \& Møller (2019). These Si and Fe dust-phase column densities are then compared against extinction, $R_{V}^{-1}$, and $c_{4}^{\prime}$ (far-UV component).

We found a strong correlation between $R_{V}^{-1}$ and $\mathrm{Si}$ and Fe dust column densities with $>96 \%$ significance. Previously, De Cia et al. (2016) found that carbonaceous grains and/or the dust grain size distribution are the main driver of the shape of the extinction curve. However, our results suggest that for featureless extinction curves, the shape of the extinction curve and dust grain size variation is linked to the amount of Si and Fe in dust-phase. High depletion of refractory elements (here $\mathrm{Si}$ and $\mathrm{Fe}$ ) leads to an increase in the fraction of large silicate grains in the ISM and vice versa. A large fraction of iron is locked as inclusions in silicate dust grains. Therefore, this correlation indicates that silicates play an important role to induce the entire extinction at any wavelength. On the contrary, the far-UV extinction curve rise is suggested to be due to the presence of small silicate grains. However, we find no trend between far-UV parameter $\left(c_{4}^{\prime}\right)$ and $\mathrm{Si}$ and Fe dust. Previously, Mishra \& Li (2017) found no trend between carbon and silicon dust trend and far-UV rise for galactic sightlines. We, therefore, suggest that far-UV extinction could be a combined effect of small grains (probably nano-sized grains) from various species.

\section{REFERENCES}

Altobelli, N., Postberg, F., Fiege, K., et al. 2016, Science, 352,312

Bilalbegović, G., Maksimović, A., \& Mohaček-Grošev, V. 2017, MNRAS, 466, L14

Bolmer, J., Ledoux, C., Wiseman, P., et al. 2019, A\&A, 623, A43

Cardelli, J. A., Clayton, G. C., \& Mathis, J. S. 1989, ApJ, 345,245

Clayton, G. C., Wolff, M. J., Sofia, U. J., Gordon, K. D., \& Misselt, K. A. 2003, ApJ, 588, 871 
Corre, D., Buat, V., Basa, S., et al. 2018, A\&A, 617, A141

Cortese, L., Ciesla, L., Boselli, A., et al. 2012, A\&A, 540, A52

Covino, S., Melandri, A., Salvaterra, R., et al. 2013, MNRAS, 432, 1231

Cucchiara, A., Fumagalli, M., Rafelski, M., et al. 2015, ApJ, 804, 51

De Cia, A. 2018, A\&A, 613, L2

De Cia, A., Ledoux, C., Mattsson, L., et al. 2016, A\&A, 596, A97

De Cia, A., Ledoux, C., Savaglio, S., Schady, P., \& Vreeswijk, P. M. 2013, A\&A, 560, A88

de Ugarte Postigo, A., Fynbo, J. P. U., Thöne, C. C., et al. 2012, A\&A, 548, A11

de Ugarte Postigo, A., Goldoni, P., Thöne, C. C., et al. 2010, A\&A, 513, A42

de Ugarte Postigo, A., Thöne, C. C., Bensch, K., et al. 2018, A\&A, 620, A190

Dorschner, J. \& Henning, T. 1995, A\&ARv, 6, 271

Draine, B. T. 1990, in Astronomical Society of the Pacific Conference Series, Vol. 12, The Evolution of the Interstellar Medium, ed. L. Blitz, 193-205

Draine, B. T. 1995, in Astronomical Society of the Pacific Conference Series, Vol. 80, The Physics of the Interstellar Medium and Intergalactic Medium, ed. A. Ferrara, C. F. McKee, C. Heiles, \& P. R. Shapiro, 133

Draine, B. T. 2003, ARAA, 41, 241

Draine, B. T. 2009, in Astronomical Society of the Pacific Conference Series, Vol. 414, Cosmic Dust - Near and Far, ed. T. Henning, E. Grün, \& J. Steinacker, 453

Draine, B. T. \& Hensley, B. 2013, ApJ, 765, 159

Draine, B. T. \& Lee, H. M. 1984, ApJ, 285, 89

Dwek, E. 2016, ApJ, 825, 136

Elíasdóttir, Á., Fynbo, J. P. U., Hjorth, J., et al. 2009, ApJ, 697, 1725

Fitzpatrick, E. L. \& Massa, D. 1990, ApJS, 72, 163

Fitzpatrick, E. L. \& Massa, D. 2007, ApJ, 663, 320

Friis, M., De Cia, A., Krühler, T., et al. 2015, MNRAS, 451, 167

Gail, H. P. 2010, Formation and Evolution of Minerals in Accretion Disks and Stellar Outflows, ed. T. Henning, Vol. 815, 61-141

Gall, C., Hjorth, J., \& Andersen, A. C. 2011, A\&ARv, 19, 43

Gioannini, L., Matteucci, F., Vladilo, G., \& Calura, F. 2017, MNRAS, 464, 985

Gordon, K. D., Clayton, G. C., Misselt, K. A., Landolt, A. U., \& Wolff, M. J. 2003, ApJ, 594, 279

Greiner, J., Krühler, T., Klose, S., et al. 2011, A\&A, 526, A30

Haenecour, P., Zhao, X., Floss, C., Lin, Y., \& Zinner, E. 2013, ApJ, 768, L17

Haris, U., Parvathi, V. S., Gudennavar, S. B., et al. 2016, AJ, 151, 143

Hartoog, O. E., Wiersema, K., Vreeswijk, P. M., et al. 2013, MNRAS, 430, 2739

Heintz, K. E., Ledoux, C., Fynbo, J. P. U., et al. 2019a, A\&A, 621, A20

Heintz, K. E., Zafar, T., De Cia, A., et al. 2019b, MNRAS, 486, 2063

Hensley, B. S. \& Draine, B. T. 2017, ApJ, 836, 179
Hilchenbach, M., Kissel, J., Langevin, Y., et al. 2016, ApJ, 816, L32

Jenkins, E. B. 1987, in Astrophysics and Space Science Library, Vol. 134, Interstellar Processes, ed. D. J. Hollenbach \& H. A. Thronson, Jr., 533-559

Jenkins, E. B. 2009, ApJ, 700, 1299

Jones, A. P. 2016, Royal Society Open Science, 3, 160221

Jones, A. P., Fanciullo, L., Köhler, M., et al. 2013, A\&A, 558, A62

Kann, D. A., Klose, S., \& Zeh, A. 2006, ApJ, 641, 993

Kemper, F., Vriend, W. J., \& Tielens, A. G. G. M. 2004, ApJ, 609, 826

Kobayashi, C. \& Nakasato, N. 2011, ApJ, 729, 16

Kobayashi, C., Umeda, H., Nomoto, K., Tominaga, N., \& Ohkubo, T. 2006, ApJ, 653, 1145

Köhler, M., Jones, A., \& Ysard, N. 2014, A\&A, 565, L9

Krühler, T., Küpcü Yoldaş, A., Greiner, J., et al. 2008, ApJ, 685, 376

Ledoux, C., Noterdaeme, P., Petitjean, P., \& Srianand, R. 2015, A\&A, 580, A8

Li, A. 2005, in Journal of Physics Conference Series, Vol. 6, Journal of Physics Conference Series, 229-248

Li, A. \& Mann, I. 2012, in Astrophysics and Space Science Library, Vol. 385, Nanodust in the Solar System: Discoveries and Interpretations, ed. I. Mann, N. Meyer-Vernet, \& A. Czechowski, 5

Lodders, K. 2003, The Astrophysical Journal, 591, 1220

Ma, J., Ge, J., Prochaska, J. X., et al. 2018, MNRAS, 474, 4870

Mathis, J. S., Rumpl, W., \& Nordsieck, K. H. 1977, ApJ, 217,425

Mautner, M. N., Abdelsayed, V., El-Shall, M. S., et al. 2006, Faraday Discussions, 133, 103

Min, M., Waters, L. B. F. M., de Koter, A., et al. 2007, A\&A, 462, 667

Mishra, A. \& Li, A. 2017, ApJ, 850, 138

Ossenkopf, V., Henning, T., \& Mathis, J. S. 1992, A\&A, 261, 567

Pei, Y. C. 1992, ApJ, 395, 130

Perley, D. A., Morgan, A. N., Updike, A., et al. 2011, AJ, 141, 36

Prochaska, J. X., Chen, H.-W., Dessauges-Zavadsky, M., \& Bloom, J. S. 2007, ApJ, 666, 267

Przybilla, N., Nieva, M.-F., \& Butler, K. 2008, The Astrophysical Journal, 688, L103

Purcell, E. M. 1969, ApJ, 158, 433

Rafelski, M., Wolfe, A. M., Prochaska, J. X., Neeleman, M., \& Mendez, A. J. 2012, ApJ, 755, 89

Sarangi, A. \& Cherchneff, I. 2015, A\&A, 575, A95

Sargent, B. A., Forrest, W. J., Tayrien, C., et al. 2009, ApJS, 182, 477

Savage, B. D. \& Sembach, K. R. 1996, ARAA, 34, 279

Savaglio, S. \& Fall, S. M. 2004, ApJ, 614, 293

Schady, P., Dwelly, T., Page, M. J., et al. 2012, A\&A, 537, A15

Schalen, C. 1965, PASP, 77, 409

Selsing, J., Malesani, D., Goldoni, P., et al. 2019, Astronomy and Astrophysics, 623, A92

Siebenmorgen, R., Voshchinnikov, N. V., \& Bagnulo, S. 2014, A\&A, 561, A82

Sofia, U. J., Wolff, M. J., Rachford, B., et al. 2005, ApJ, 625,167 
Stecher, T. P. 1965, ApJ, 142, 1683

Tielens, A. G. G. M. \& Allamandola, L. J. 1986, Composition, structure, and chemistry of interstellar dust., Tech. rep.

Tinsley, B. M. 1979, ApJ, 229, 1046

Valencic, L. A., Clayton, G. C., \& Gordon, K. D. 2004, ApJ, 616, 912

Valencic, L. A., Clayton, G. C., Gordon, K. D., \& Smith, T. L. 2003, ApJ, 598, 369

Vladilo, G., Abate, C., Yin, J., Cescutti, G., \& Matteucci, F. 2011, A\&A, 530, A33

Vladilo, G., Centurión, M., Levshakov, S. A., et al. 2006, A\&A, 454, 151

Wang, S., Li, A., \& Jiang, B. W. 2015, ApJ, 811, 38

Weingartner, J. C. \& Draine, B. T. 2001, ApJ, 548, 296

Westphal, A. J., Stroud, R. M., Bechtel, H. A., et al. 2014, Science, 345, 786

Wiseman, P., Schady, P., Bolmer, J., et al. 2017, A\&A, 599, A24

Zafar, T., Heintz, K. E., Fynbo, J. P. U., et al. 2018a, ApJL, 860, L21

Zafar, T. \& Møller, P. 2019, MNRAS, 482, 2731

Zafar, T., Møller, P., Ledoux, C., et al. 2011a, A\&A, 532, A51

Zafar, T., Møller, P., Watson, D., et al. 2018b, MNRAS, 480, 108

Zafar, T. \& Watson, D. 2013, A\&A, 560, A26

Zafar, T., Watson, D., Elíasdóttir, Á., et al. 2012, ApJ, 753, 82

Zafar, T., Watson, D., Fynbo, J. P. U., et al. 2011b, A\&A, 532, A143

Zafar, T., Watson, D., Møller, P., et al. 2018c, MNRAS, 479,1542

Zhukovska, S., Dobbs, C., Jenkins, E. B., \& Klessen, R. S. 2016, ApJ, 831, 147

Zhukovska, S., Henning, T., \& Dobbs, C. 2018, ApJ, 857, 94

Zubko, V. G., Smith, T. L., \& Witt, A. N. 1999, ApJ, 511, L57

This paper has been typeset from a $\mathrm{T}_{\mathrm{E}} \mathrm{X} / \mathrm{HT}_{\mathrm{E}} \mathrm{X}$ file prepared by the author. 